\title{
Relación entre el factor agua/cemento, la resistencia normalizada del cemento (DIN 1 164, junio 1970) y la resistencia del hormigón a la compresión \\ KURT WALZ, Profesor Dr. Ingeniero \\ Beton, no 11, noviembre 1970, págs. 499-503
}

\section{R E S U M E N}

Con arreglo a la nueva norma de ensayos de cemento DIN 1164 (junio 1970), los cementos se ensayan según un método algo distinto al anteriormente existente. Este nuevo método de ensayo corresponde a la recomendación ISO $\mathrm{R}$ 679. Se obtiene así, para un mismo cemento, una resistencia normalizada a la compresión generalmente un poco más elevada.

Los diagramas publicados hace unos 15 años que relacionan el factor agua/cemento de los hormigones, la resistencia normalizada a la compresión del cemento y la resistencia a la compresión de un cubo normalizado de hormigón a la edad de 28 días, fue preciso rehacerlos. Con ellos puede proyectarse nuevamente la composición de una mezcla de hormigón o estimarse la resistencia a la compresión previsible.

Como base de los diagramas sirvieron los resultados de los ensayos de unas 830 mezclas de hormigón que se hicieron en el laboratorio desde 1963.

\section{GENERALIDADES}

Para proyectar una mezcla de hormigón con la que se ha de conseguir una resistencia a la compresión de un cubo normalizado después de 28 días, mediante fabricación, almacenamiento y ensayo según DIN 1 048, se emplean, frecuentemente, fórmulas o diagramas. Se basan, la mayoría de las veces, en una relación entre la resistencia a la compresión del hormigón, la resistencia normalizada del cemento a emplear y la relación agua/cemento del hormigón fresco. La figura 1 reproduce esquemáticamente uno de estos diagramas. Se reconoce la dependencia de la resistencia a la compresión del hormigón $\beta_{\mathrm{D}}$ de la relación agua/cemento $\mathrm{w}$ (esta es la relación del peso de todo el agua $\mathrm{W}$, incluida humedad superficial del árido, al peso del cemento $\mathrm{Z}$ en $1 \mathrm{~m}^{3}$ del hormigón fresco compactado, prácticamente, en su totalidad). A igualdad de otras cosas, la resistencia a compresión del hormigón se reduce al elevar la relación agua/cemento, según una curva continua. O sea, que la resistencia a la compresión es tanto más reducida cuanto más agua 
contenga la pasta de cemento, es decir, cuanto más fluida sea (ley de la relación agua/cemento).

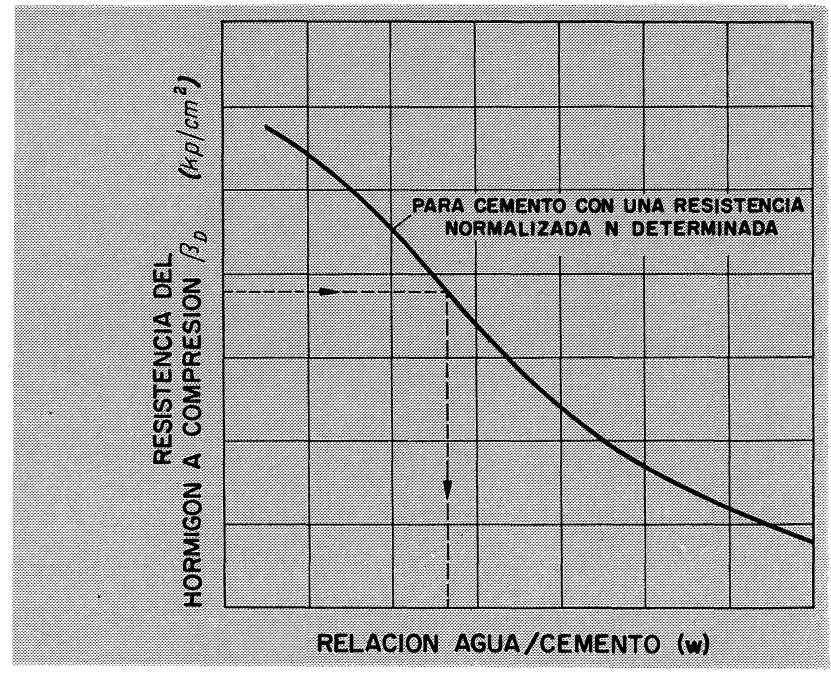

Fig. 1.-Relación (esquemática) entre la relación agua/cemento $w$, la resistencia a compresión normalizada de los cementos $\mathbf{N}$ y la resistencia a compresión del hormigón $\beta_{D}$.

relación agua/cemento y la resistencia normal del cemento a la compresión.

Un diagrama que se utiliza a menudo (véase, por ejemplo [1]), se basa, con arreglo a la versión antigua de la DIN 1164 (última edición, diciembre 1958), en la resistencia normal del cemento a la compresión después de 28 días; el mortero de ensayo tiene una relación agua/cemento de 0,60. De acuerdo con la versión nueva de la DIN 1 164, edición junio 1970, los cementos se han clasificado en otros tipos de resistencia con limitación inferior y superior de la resistencia normal a la compresión después de 28 días. Su resistencia normal se determina, según la hoja 7 de la norma, con un método de prueba modificado y, sobre todo, con una relación agua/cemento de 0,50. Este ensayo corresponde a las pruebas del cemento con arreglo al "RILEM-Cembureau-Method" (ISO Recommendation $\mathrm{R}$ 679: Method of testing strength of cements; marzo 1968).

Como quiera que la ley de la relación agua/cemento también repercute en el mortero de ensayo aumentando la resistencia al disminuir la relación agua/cemento, con el ensayo, según la nueva norma, para un cemento determinado se obtiene distinta resistencia normal a la compresión, algunas veces algo menor o igual, pero, generalmente, algo mayor que con el método de ensayo anterior (véase también [2]). Esto sólo significa resistencia específica de un cemento (resistencia normalizada), que ahora se caracteriza con una medida algo distinta. Además, este mortero de ensayo con menor relación agua/cemento y una arena de granulometría más amplia, con cementos molidos más gruesos tiende a segregar menos el agua que el empleado hasta ahora. Con mucha segregación

(1) Para más detalle sobre este punto, principalmente teniendo en cuenta la DIN 1045 (1971), véase Walz, K.: Fabricación de hormigón según DIN 1045, Beton-Verlag, Düsseldorf (en preparación). 
del agua, en casos extremos, el actual mortero de ensayo en vez de con una relación agua/cemento de 0,60 podía endurecerse con una de sólo 0,50; acusaba así una mayor resistencia normal, que como tal o como "rendimiento" del cemento no debe repercutir sin más en el hormigón. Este es el caso cuando el hormigón endurece sin la correspondiente segregación de agua, por ejemplo cuando presenta elevado contenido de finos o contiene determinados aditivos. Es decir, el "rendimiento" de los cementos se determina más uniforme y acertadamente mediante el nuevo método de ensayo.

De esto se deduce que con relaciones agua/cemento $\mathrm{w}$ iguales de un hormigón, con el nuevo método de ensayo del cemento varía también la relación entre la resistencia a compresión del hormigón $\beta_{\mathrm{D} 28}$ y la resistencia normal a compresión $\mathrm{N}_{28}$ del cemento ${ }^{(2)}$. Por lo tanto, en lugar del diagrama anterior [1] se confeccionó un nuevo diagrama. Este fue el resultado de la valoración de unas 830 mezclas de hormigón con cementos cuya resistencia normal a la compresión $\mathrm{N}_{28}$ se determinó según DIN 1164, edición junio 1970.

\section{RELACIONES ENTRE $\boldsymbol{w}, \mathbf{N}_{28}$ y $\beta_{\mathrm{D} 28}$}

\subsection{Documentación}

Para la confección de los antiguos diagramas se emplearon mezclas de ensayo de hormigón del Instituto Otto-Graf de la Universidad de Stuttgart, hasta aproximadamente el año 1955. El nuevo diagrama se basa en unas 290 mezclas, fabricadas en la sección técnica del hormigón del Instituto de Investigaciones de la Industria del Cemento en Düsseldorf. Van hacia atrás hasta el año 1963, a partir del cual se han ensayado todos los cementos con arreglo a un nuevo método normalizado. Además, se han incluido resultados de ensayos de una parte de los trabajos tecnológicos del hormigón que realizaron estudiantes en escuelas de ingenieros con apoyo de la Asociación de fábricas alemanas de cemento desde 1963 (unas 540 mezclas de ensayo).

La consistencia de las mezclas cubrió la zona de la DIN 1045 (1971).

El árido, predominantemente grava con un tamaño máximo de $30 \mathrm{~mm}$, procedía de diversas comarcas de la República Federal. Los 78 cementos empleados (portland, portland siderúrgico y portland de horno alto) se distinguían por su procedencia, fecha de suministro y tipo de resistencia; predominaban los cementos portland. La resistencia normal $\mathrm{N}_{28}$ se fijó en el ensayo entre unos 360 y $700 \mathrm{kp} / \mathrm{cm}^{2}$.

La relación agua/cemento de las mezclas de hormigón se encontraba entre aproximadamente 0,35 y 1,20 , pero predominantemente entre 0,40 y 0,80 . (El número de mezclas de las que se disponía para las diversas relaciones agua/cemento, se indica en la parte superior de la figura 2, apartado 2.2). El contenido de cemento iba de unos 150 hasta 500 $\mathrm{kg} / \mathrm{m}^{3}$.

Las curvas granulométricas de las mezclas de áridos eran continuas y acompasadas a

(2) La resistencia a la compresión de un cubo normalizado de hormigón determinada en el diagrama y a justificar posteriormente mediante la prueba de calidad a la edad de 28 días se designó con $\beta_{\mathrm{D} 28}$, a fin de evitar confusiones con las designaciones utilizadas en la prueba de calidad de la tabla 1 de la DIN 1045 (1971). Allí, $\beta_{\text {w28 }}$ es la resistencia mínima a la compresión de cada uno de los cubos o bien $\beta_{w M}$ es la resistencia mínima a la compresión del valor medio de cada serie de 3 cubos que proceden de diferentes mezclas. 
las curvas granulométricas límite D, E. y F. representadas en la figura 2 de la DIN 1045 (antigua), y frecuentemente a la zona prácticamente más usada entre D/E a E/F.

La resistencia a la compresión del hormigón $\beta_{\mathrm{D}_{28}}$ es el promedio de 3 cubos de una mezcla de $20 \mathrm{~cm}$ de arista. Los cubos se han fabricado según DIN 1048 (prácticamente compactados en su totalidad) y ensayados según ella. Se almacenaron en húmedo durante 7 días y a continuación al aire ambiente. En el Instituto de Investigaciones, la temperatura del local de conservación era uniformemente de $20^{\circ} \mathrm{C} \pm 2$ grados y la humedad relativa del aire durante la conservación ambiente de $65 \% \pm 2 \%$. Por los análisis efectuados en las escuelas de ingenieros, puede admitirse que en la conservación la temperatura ambiente se ha mantenido entre $15^{\circ}$ y $22^{\circ} \mathrm{C}$ prescrita en DIN 1048.

Las resistencias a la compresión encontradas para los cubos normalizados de hormigón $\beta_{\mathrm{D}_{28}}$ mostraban valores extremos entre unos 110 y $730 \mathrm{kp} / \mathrm{cm}^{2}$ y, en su mayor parte, entre unos 200 y $550 \mathrm{kp} / \mathrm{cm}^{2}$.

\subsection{Confección del diagrama}

Todos los datos de las mezclas fueron pasados a fichas perforadas ${ }^{(3)}$, y para cada mezcla se formó la relación $\beta_{\mathrm{D}_{28}} / \mathrm{N}_{28}$. El computador electrónico determinó el valor medio correspondiente a la relación $\beta_{\mathrm{D}_{28}} / \mathrm{N}_{28}$ y la diferencia $s$ constreñidos a las relaciones agua/cemento en 0,05 . Para las relaciones agua/cemento $0,40,0,50,0,60,0,70$ y 0,80 se disponía entre 106 y 175 valores individuales de $\beta_{\mathrm{D}_{28}} / \mathrm{N}_{28}$, y para los intermedios, de 26 a 47. Del valor medio y la desviación standard se ha calculado la fractila superior e inferior de $5 \%$.

Los valores medios de $\beta_{\mathrm{D}_{28}} / \mathrm{N}_{28}$ y las fractilas de $5 \%$ se incluyeron en un diagrama como ordenadas frente a las relaciones agua/cemento como abscisas; formaron los puntos de referencia de curvas de nivel. El diagrama resultante se reproduce en la figura 2.

La desviación standard calculada por las curvas de nivel $s$ se encuentra entre 24 y 80 $\mathrm{kp} / \mathrm{cm}^{2}$ según la magnitud de la resistencia a la compresión $\beta_{\mathrm{D} 28}$ (dependiente de $\mathrm{w} \mathrm{y}$ de $\mathrm{N}_{28}$ ). A mayor resistencia a la compresión se dan desviaciones standard mayores, aunque, desde luego, no aumentaron en igual medida que la resistencia, de tal forma que el coeficiente de variación $v$ disminuyó de $16 \%$ a aproximadamente $11 \%$ al crecer la resistencia a la compresión $\beta_{\mathrm{D}_{28}}$. Individualmente se encontró para:

$$
\begin{aligned}
& \beta_{\mathrm{D}_{28}}=150 \quad 200 \quad 300 \quad 400 \quad 500 \quad 600 \quad 700 \quad \mathrm{kp} / \mathrm{cm}^{2} \\
& s=\begin{array}{llllllll}
24 & 32 & 43 & 50 & 58 & 69 & 80 & \mathrm{kp} / \mathrm{cm}^{2}
\end{array} \\
& v=\quad \begin{array}{llllllll}
16,0 & 16,0 & 14,3 & 12,5 & 11,6 & 11,5 & 11,4 & \%
\end{array}
\end{aligned}
$$

De las relaciones de la figura 2 se derivó otro diagrama, figura 3 , que permite partir directamente de la resistencia del hormigón a la compresión $\beta_{\mathrm{D}_{28}}$ prevista y de los tipos de resistencia Z 250, Z 350, Z 450 y Z 550 del cemento y leer la correspondiente relación agua/cemento w. El diagrama se basa en las siguientes resistencias normalizadas medias a la compresión $\mathrm{N}_{28 \mathrm{M}}$ para los 4 tipos de resistencia: $\mathrm{N}_{28 \mathrm{M}}=350 \mathrm{kp} / \mathrm{cm}^{2}$ para $\mathrm{Z} 250$, $\mathrm{N}_{28 \mathrm{M}}=450 \mathrm{kp} / \mathrm{cm}^{2}$ para Z $350, \mathrm{~N}_{28 \mathrm{M}}=550 \mathrm{kp} / \mathrm{cm}^{2}$ para Z $450 \mathrm{y} \mathrm{N}_{28 \mathrm{M}}=635 \mathrm{kp} / \mathrm{cm}^{2} \mathrm{pa}-$ ra Z 550 .

(3) En la valoración me ha apoyado el Dipl.-Ing. Sr. Dartsch, colaborador en la sección técnica del hormigón del Instituto de Investigaciones. Las sugerencias y el examen del original manuscrito se lo agradezco a los Srs. Dr. Bonzel, Prof. Locher y Dr. Wischers. 
Se partió aquí del hecho de que los cementos según DIN 1164, hoja 1 (edición junio 1970), se dividen en 4 tipos resistentes:

\section{Z 250, Z 350, Z 450 y Z 550.}

Estas cifras indican la resistencia mínima a la compresión $\mathrm{N}_{28}$ en $\mathrm{kp} / \mathrm{cm}^{2}$, no debiendo quedar debajo de ella en ningún ensayo individual. Además, la resistencia normalizada a la compresión determinada en cada ensayo individual, en los tres tipos inferiores, sólo será, como máximo, $200 \mathrm{kp} / \mathrm{cm}^{2}$ superior a la resistencia normalizada mínima a la compresión. En el tipo superior Z 550, debido al estado actual de la técnica del cemento se establece automáticamente un límite superior de resistencia similar.



Fig. 2.-Relación entre el factor agua/cemento $\mathrm{w}$, la resistencia a compresión normalizada de los cementos $\mathbf{N}_{28}$ (norma DIN 1164 , edición junio 1970) y la resistencia a compresión en cubos de los hormigones $\beta_{\mathrm{D} 28}$ (relación media empírica de 830 mezclas de hormigón obtenidas en el laboratorio).

Fig. 3.-Dependencia de la resistencia a compresión de los cilindros de hormigón $\beta_{\mathrm{D} 28}$ de la relación agua/cemento y del tipo resistente de los cementos según DIN 1 164, edición junio 1970 (deducida de la relación obtenida en la figura 2 con las resistencias normalizadas a compresión subsiguientes: $\mathbf{N}_{28 \mathrm{M}}=350 \mathrm{kp} / \mathrm{cm}^{2}$ para $Z 250, N_{28 M}=450 \mathrm{kp} / \mathrm{cm}^{2}$ para $Z 350$

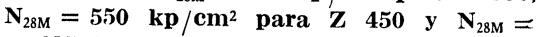
$=635 \mathrm{kp} / \mathrm{cm}^{2}$ para $\mathrm{Z} 550$ ).

De la gama de resistencias $\left(200 \mathrm{kp} / \mathrm{cm}^{2}\right)$ admitida para los tres tipos inferiores puede sacarse la consecuencia de que la resistencia de los distintos suministros de cemento pue- 
den fluctuar entre estos límites. Pero no es éste el caso, porque estos valores no deben de quedar nunca por debajo ni por encima en ningún ensayo individual, y tampoco teniendo en cuenta las dispersiones de los ensayos, cuya desviación standard se halla en el orden de por lo menos $20 \mathrm{kp} / \mathrm{cm}^{2}$. Con arreglo a análisis estadísticos, en cada caso sólo el $5 \%$ de los suministros divergirán en más de $\pm 50 \mathrm{kp} / \mathrm{cm}^{2}$ del valor medio ajustado, y unos $2 / 3$ de todos los valores de los ensayos caerán en una zona que en cuanto al orden de magnitud sólo difiere $\pm 30 \mathrm{kp} / \mathrm{cm}^{2}$ del valor medio. $O$ sea, que está justificado el que para proyectar mezclas de hormigón con cementos de los tipos resistentes Z 250, Z 350 y Z 450 se elija una resistencia normal a compresión que se encuentre en el centro entre las resistencias límites inferiores y superiores en cada caso, es decir, $100 \mathrm{kp} / \mathrm{cm}^{2}$ sobre la resistencia normalizada mínima a la compresión.

\section{EMPLEO DE LA RELACION}

\subsection{Magnitudes influyentes}

La relación $\mathrm{w} / \mathrm{N}_{28} / \beta_{\mathrm{D} 28}$ sirve para condiciones medias. O sea, que en ella aparece la relación agua/cemento juntamente con la resistencia normal del cemento como factores influyentes y decisivos en la resistencia del hormigón $\beta_{\mathrm{D}_{28}}$. Además pueden influir otras circunstancias, aunque no sean decisivas; cuantitativamente no pueden abarcarse fácilmente y, por lo tanto, parece prudente no exigir en general una exactitud exagerada en las relaciones $\mathrm{w} / \mathrm{N}_{28} / \beta_{\mathrm{D}_{28}}$. Individualmente hay que observar a este respecto lo siguiente:

3.1.1. La relación agua/cemento, puesta aquí como base, se refiere a todo el agua del hormigón fresco. Con arreglo a los fundamentos teóricos de la ley de la proporción agua/ /cemento, se parte de que en la pasta de cemento del hormigón quedan, en cada momento del endurecimiento, poros capilares que disminuyen la resistencia, tanto más llenos de agua cuanto mayor fue el contenido de agua del hormigón. En la relación agua/cemento de las expresiones establecidas en las figuras 1,2 y 3 se incluye también una parte del agua $\mathrm{W}$, que en su reacción con el cemento (hidratación) se incorpora química y físicamente en los productos de la reacción (formaciones nuevas). Este agua no coadyuva a la formación de poros; además, las nuevas formaciones que condicionan la resistencia ocupan un espacio aproximadamente doble de grande que la parte del cemento que se hidrató hasta un momento determinado. Es decir, que el espacio de poros capilares que disminuye la resistencia y que primeramente está lleno de agua, es, en la pasta de cemento menor que el contenido de agua $\mathrm{W}$ indicado para la proporción de agua/cemento del hormigón fresco (pasta de cemento). La cantidad de agua que se fija por ejemplo hasta la edad de 28 días, depende de la capacidad de reacción del cemento y de la temperatura de endurecimiento. Cementos que reaccionan con mucha rapidez, como son los del tipo resistente $\mathrm{Z} 550$, se han hidratado ampliamente a los 28 días con suficiente fijación de agua ; otros precisan de más tiempo. Puede suponerse que, por ejemplo, los cementos normalizados del tipo Z 350 , hasta la edad de 28 días, únicamente fijan de 60 a $80 \%$ de la cantidad de agua que se precisa para la completa hidratación.

A grandes rasgos, los cementos normalizados necesitan para su hidratación completa de 37 a $40 \%$ de peso de agua, lo cual corresponde a una relación agua/cemento de aproximadamente 0,40 . Con éstas y, naturalmente, también con relaciones agua/cemento menores, el espacio ocupado primitivamente por el agua en la pasta de cemento lo rellenan 
las nuevas formaciones sólidas, de forma que ya no queda ningún hueco de poros capilares ${ }^{(4)}$.

Basta hacer constar aquí que todos los cementos en su endurecimiento, hasta la edad que aquí interesa de 28 días, fijan fuertemente una parte determinada del agua de amasado y, en consecuencia, por las nuevas formaciones el espacio de poros capilares que procede de la totalidad del agua de amasado en la pasta de cemento primitiva disminuye correspondientemente más o menos. $\mathrm{O}$ sea, que en realidad debiera tenerse únicamente en cuenta la cantidad de agua que forma los poros capilares, lo cual depende de la relación agua/cemento global y del grado de hidratación del cemento. La influencia del grado de hidratación de un cemento se la tiene ampliamente en cuenta al incluir la resistencia normal del cemento, ya que en el mortero de ensayo normalizado con una relación agua/cemento de 0,50 tiene lugar, hasta la edad de 28 días, una fijación de agua que depende de las características de endurecimiento del conglomerante. Pero esta fijación del agua, para unas relaciones agua/cemento del hormigón distintas de 0,50, puede ser algo diferente de la que tiene lugar en el mortero normalizado. Hay que suponer además que en la dispersión también coadyuva la distinta conservación del mortero normalizado (bajo agua) y del hormigón (al aire). A consecuencia de todo ello, en función del tipo y clase de resistencia de los diversos cementos, la resistencia del hormigón puede desarrollarse en forma algo distinta que como se indica por la resistencia normal del cemento.

A continuación se mencionan otras causas que actúan en la dispersión.

3.1.2. En varios trabajos se ha hecho constar que, a igualdad de relación agua/cemento, la resistencia a la compresión de hormigón con altos contenidos de cemento (por ejemplo más de $350 \mathrm{~kg} / \mathrm{m}^{3}$ ) al crecer el volumen de pasta ya no actúa totalmente de acuerdo con dicha proporción agua/cemento. Esta influencia se manifiesta probablemente en la figura 2, al observarse al principio de la curva un recorrido más plano hasta la zona de la relación agua/cemento de 0,40. (La mayoría de las mezclas empleadas con proporciones agua/cemento inferiores a 0,40 tenían contenidos de cemento de más de $350 \mathrm{~kg} / \mathrm{m}^{3}$ ). El volumen de pasta de cemento en tales hormigones es grande, y entre los granos gruesos del árido existen capas gruesas de pasta de cemento o mortero fino. La menor eficacia del conglomerante en un hormigón muy rico en cemento puede explicarse por el hecho de que para capas espesas entre los gránulos más gruesos es decisiva, en el esfuerzo de compresión, una especie de "resistencia de un prisma", que es notablemente menor que la "resistencia de una placa" de capas más delgadas entre los granos del árido con contenidos menores de cemento. (La resistencia específica a la compresión de la pasta de cemento o del mortero fino es, naturalmente, de igual magnitud en ambos casos).

Una influencia en el mismo sentido es también la menor superficie del árido en un hormigón con elevado contenido de cemento, que para el sistema "pasta de cemento-árido"

\footnotetext{
(4) A pesar de que con relaciones agua/cemento menores que aproximadamente 0,40 ya no todo el cemento crea nuevas formaciones y ya en la relación agua/cemento 0,40 no quedan más poros capilares, a relación agua/cemento decreciente surge todavía un aumento de resistencia. (Esta zona de relaciones agua/cemento no tiene aquí ninguna importancia, ya que un hormigón fresco con semejantes proporciones agua/cemento, en las condiciones constructivas prácticas, es, en general, demasiado rígido para una compactación total). Para esto hay varias explicaciones. Entre otras, puede suponerse que los núcleos no hidratados de los pequeños gránulos de cemento, más correctamente granulitos de clínker, que quedan entre las formaciones nuevas, son más sólidos que éstas (gel de cemento); así hacen rígidas las nuevas formaciones actuando a modo de una armadura de áridos; véase también su analogía con el hormigón en 3.1.2. y otras consideraciones en [3]. Además, bajo estas condiciones se separa una cantidad menor de hidróxido cálcico, que aporta poco a la resistencia de las nuevas formaciones [4].
} 
significa menos superficie de adherencia en la transmisión de esfuerzos a los más sólidos gránulos de la piedra y mayor discontinuidad de la estructura con diversas repercusiones desfavorables.

En correspondencia, también se ofrece una explicación para algunas determinaciones, según las cưales, con mezclas de árido más finas, igual cantidad de cemento y la misma proporción agua/cemento se consiguen resistencias a la compresión algo mayores que con mezclas de áridos más gruesos.

3.1.3. El hormigón de los cubos se compactó "prácticamente por completo". En tal hormigón existe todavía, en muchos casos, un contenido de aire ocluido superior al 1,5\% en volumen. $\mathrm{Si}$, por ejemplo, se incluye más aire debido al empleo de aditivos, se hace mayor el espacio de los poros y se le considerará como reductor de resistencias (véase también párrafo 3.2.3.). Con aditivos, por tanto, el curso del desarrollo de la resistencia en el hormigón puede ser algo distinto del caracterizado por la resistencia normalizada del cemento.

3.1.4. También la distinta adherencia entre superficie de las piedras y la pasta de cemento, consecuencia de la textura superficial de los gránulos del árido y su composición mineralógica, repercute en la resistencia del hormigón a la compresión.

Ademas, en función de la capacidad de fijación de agua del árido, se puede reducir algo la proporción agua/cemento empleando árido seco, lo cual produce un aumento de la resistencia a compresión del hormigón en la zona superior de dispersión. A la inversa, la adherencia de un árido saturado de agua habrá que suponerla algo más desfavorable que la de uno que todavía puede absorber algo de agua.

3.1.5. En función del cemento, la resistencia a compresión del hormigón puede resultar algo distinta a pesar de la conservación de acuerdo con las normas, según que la temperatura de fabricación y conservación de los cubos se encuentre dentro de la zona de $+15^{\circ} \mathrm{a}+22^{\circ} \mathrm{C}$ fijada en DIN 1048 , en el límite inferior o en el superior.

El curso del endurecimiento puede también, al principio, estar influenciado de distinto modo y diferir del mortero normalizado, según que el hormigón, en función del tipo y contenido de cemento, desarrolle en el molde más o menos calor. A esto hay que añadir que, según DIN 1048, el almacenamiento inicial de 7 días en húmedo puede hacerse bajo agua o en arena húmeda. La humedad del hormigón en el ensayo, después de la conservación posterior de 21 días al aire, puede ser distinta y, en determinadas circunstancias, influir algo en el resultado del ensayo de resistencia a la compresión (con alto contenido de humedad aparece una resistencia a la compresión algo menor que la que corresponde al grado de endurecimiento propiamente dicho; véase también [5]). Esto se debe a que tampoco en DIN 1048 se establece la humedad relativa del aire en el local de conservación.

Las influencias que se han tratado aquí corresponden a la denominada dispersión de fabricación y ensayo; a éstas hay que añadir todavía otras, debidas, por ejemplo, a un dimensionado inexacto, a distintos mezclados y compactado de las mezclas de ensayo, a superficies de compresión irregulares, a distinta velocidad de ensayo, etc.

3.1.6. Resumiendo, debe decirse que por una serie de influencias es inevitable una zona de dispersión en la relación $\mathrm{w} / \mathrm{N}_{28} / \beta_{\mathrm{D}_{28}}$ de la figura 2, caracterizada por la fractila de 5 $\%$. Esta curva media, obtenida por una multitud de mezclas de ensayo para la relación $\mathrm{w} / \mathrm{N}_{28} / \beta_{\mathrm{D} 28}$, proporciona, sin embargo - como muestra la experiencia con la curva an- 
terior [1] - , una buena base para el proyecto o el dictamen de mezclas en una zona que en la práctica aparece como la más frecuente ${ }^{(5)}$.

Una mejor comprensión de la fluencia de los materiales que se presentan en cada caso y una comprobación del proyecto de mezcla basado en condiciones medias y que se ha elaborado según las figuras 2 ó 3 , es posible con la prueba de calidad que según DIN 1045 ha de seguir a un proyecto de mezcla.

\subsection{Prueba de calidad}

3.2.1. Con arreglo a la norma DIN 1045 (1971), la prueba de calidad sirve para determinar, previamente, qué composición ha de tener el hormigón para que pueda ser elaborado con garantía con las sustancias de partidas propuestas y la consistencia prevista en las condiciones de la obra correspondiente y consiga con seguridad las propiedades requeridas. Se indicará además, por separado, en qué circunstancias son necesarias pruebas de calidad, fijándose también la proporción agua/cemento precisa.

En la prueba de calidad, el valor medio de la resistencia a la compresión de los 3 cubos de la mezcla de hormigón cuya composición ha de ser determinante para la ejecución de la obra, incluirá un cierto margen de seguridad. Este será, de acuerdo con la resistencia media a la compresión mínima a garantizar en la prueba de calidad, $\beta_{\mathrm{wM}}{ }^{(2)}$ para los tipos de resistencia (resistencia nominal) Bn 100 hasta Bn 250 inclusive (hormigón B I) de por lo menos $50 \mathrm{kp} / \mathrm{cm}^{2}$; para hormigón B II (tipos de resistencia Bn 350 y superiores), el margen puede elegirse teniendo en cuenta la zona de dispersión que, según la experiencia, es de esperar en la obra correspondiente. Como quiera que la resistencia media a la compresión $\beta_{\mathrm{wM}}$ a conseguir como mínimo ya se ha puesto $50 \mathrm{kp} / \mathrm{cm}^{2}$ por encima de la resistencia nominal $\beta_{\mathrm{wN}}$ (tipo de resistencia del hormigón $\mathrm{Bn}$ ), esto significa que en la prueba de calidad para hormigón I el resultado del ensayo de la compresión ha de estar $100 \mathrm{kp} / \mathrm{cm}^{2}$ por encima de la resistencia nominal y para el hormigón II $50 \mathrm{kp} / \mathrm{cm}^{2}$ sobre la resistencia nominal, incorporando unos razonables márgenes de seguridad a elegir. Por consiguiente, la relación agua/cemento necesaria se tomará de las figuras 2 ó 3 , para una resistencia a la compresión $\beta_{\mathrm{D}_{28}}$ que tenga en cuenta estas condiciones.

3.2.2. En una prueba de calidad se utilizarán 3 mezclas, que se diferencian de la mezcla derivada de las figuras 2 ó 3 y calculada, según el apartado $1,2^{\circ}$ párrafo [8], en relaciones agua/cemento en, aproximadamente, $+0,05 \mathrm{y}-0,05$. Es de esperar que así se cubran las influencias adicionales (véase apartado 3.1.) y que la mezcla de obra pueda establecerse definitivamente con arreglo al resultado de estas 3 mezclas o que, en caso necesario, sea factible una corrección sencilla de la mezcla calculada.

3.2.3. Como se cita en el párrafo 3.1.3., las figuras 2 ó 3 son válidas para hormigones con un contenido natural de oclusiones de aire de aproximadamente $1,5 \%$. Con mayores contenidos de aire debidos a la incorporación de aditivos en el hormigón, el contenido de

\footnotetext{
(5) En la norma ACI 613-54, revisión de 1969: "Recommended practice for selecting proportions for normal weight concrete" [6] se encuentra la tabla 5.2.4. (a), en la que se indica la proporción agua/ /cemento necesaria $(0,82$ a 0,41$)$ para obtener resistencias a la compresión de un cubo normalizado de hormigón de 180 a $540 \mathrm{kp} / \mathrm{cm}^{2}$ (convertido según [7]). Estas resistencias a la compresión coinciden muy bien con la relación de la figura 3 para proporciones agua/cemento de 0,45 a 0,75 , si se utiliza la curva para la resistencia normalizada media del $\mathrm{Z} 350\left(\mathrm{~N}_{28 \mathrm{M}}=450 \mathrm{kp} / \mathrm{cm}^{2}\right)$.
} 
aire L que excede de esta cifra hay que incluirlo - puesto que reduce la resistencia - como una parte del agua de amasado.

3.2.4 Para el proyecto de mezclas de hormigón a las que se exige una determinada resistencia a la compresión $\beta_{\mathrm{D}}$ antes de los 28 días, con las resistencias normalizadas del cemento, según el nuevo método de ensayo, todavía no se dispone de ninguna relación de acuerdo con las figuras 2 ó 3 . Como se puede reconocer al valorar algunos ensayos ([9] y [10]), para las variables $\mathrm{w}, \mathrm{N}$ y $\beta_{\mathrm{D}}$, a edades inferiores (por ejemplo, 3 ó 7 días), se producen resistencias bajas del hormigón, referidas a la edad de 28 días, con valores medios que se relacionan bien con la resistencia normalizada a la edad correspondiente. Por lo tanto, al proyectar una mezcla de hormigón para una resistencia dada a baja edad puede utilizarse como base aproximada, y con ciertas limitaciones para caso individual, la relación de acuerdo con la figura 2, siempre que para $\mathrm{N}$ se tome la resistencia normalizada del cemento a la edad correspondiente.

\section{RESUMEN}

4.1, Los diagramas utilizados hasta ahora, basados en la relación entre proporción agua/ /cemento $\mathrm{w}$, resistencia normalizada a la compresión del cemento $\mathrm{N}_{28}$ y la resistencia (normalizada) a la compresión de un cubo normalizado de hormigón $\beta_{\mathrm{D}_{28}}$, han demostrado ser útiles para proyectar mezclas de hormigón al que a los 28 días se le exige una determinada resistencia a la compresión en forma de cubo normalizado $\beta_{\mathrm{D}_{28}}$, o para calcular la resistencia prevista a la compresión si son conocidos w y $\mathrm{N}_{28}$.

4.2. Como quiera que en el futuro el cemento se ensayará de acuerdo con la norma DIN 1164 , edición junio 1970, con un mortero normalizado que presenta una relación agua/cemento de 0,50 frente a la anterior de 0,60, en el ensayo del cemento se obtiene ahora, generalmente, una resistencia normal a la compresión algo mayor $\mathrm{N}_{28}$. Es natural que a consecuencia de esto no sea distinta la resistencia a la compresión de un cubo normalizado que se espera para una determinada mezcla de hormigón; sin embargo, la relación existente $\mathrm{w} / \mathrm{N}_{28} / \beta_{\mathrm{D}_{28}}$ no puede utilizarse ya para el proyecto de mezclas debido a la magnitud variable de $\mathrm{N}_{28}$.

4.3. Se ha establecido una nueva relación en forma de diagrama (figura 2) mediante el ensayo de unas 830 mezclas de hormigón hechas en el laboratorio. Para las mezclas se emplearon numerosos cementos, cuya resistencia normalizada $N_{28}$ se ha determinado con arreglo a la nueva DIN 1164 . La composición de los hormigones fue muy variada y cubrió toda la gama de mezclas que se presentan en la práctica en cuanto a contenido de cemento, relación agua/cemento, consistencia y curvas granulométricas del árido con $30 \mathrm{~mm}$ de tamaño máximo. La resistencia a la compresión $\beta_{\mathrm{D}_{28}}$ sirve para cubos como los que se han de confeccionar, conservar y ensayar, en la prueba de calidad según DIN 1048 .

Se estableció, derivado del anterior, un segundo diagrama (figura 3) en el que se incluyen 4 curvas de referencia, válidas para una resistencia normalizada media de los 4 tipos resistentes de los cementos Z 250, Z 350, Z 450 y Z 550 fijados en la nueva norma.

Lo mismo que los diagramas antiguos, los nuevos diagramas se obtuvieron empíricamente; reproducen en curvas la relación media $\mathrm{w} / \mathrm{N}_{28} / \beta_{\mathrm{D}_{28}}$. La dispersión alrededor de ellas es consecuencia de una serie de influencias debidas a las peculiaridades de la relación, a las características de las diversas mezclas y al propio ensayo. 
4.4. Con la proporción agua/cemento obtenida por la relación media de los diagramas, con un contenido de agua calculado en el hormigón fresco puede determinarse el correspondiente contenido de cemento y, por cálculo del espacio que ocupa el material, establecerse la proporción de áridos. Este sencillo cálculo poco costoso puede realizarse sin diagramas complementarios y permite así tener también en cuenta en cada caso otros factores influyentes secundarios, así como profundizar en el conocimiento de las relaciones que han de observarse en general al proyectar mezclas de hormigón.

La mezcla proyectada se pone como base para la prueba de calidad exigida en DIN 1045.

\section{B I B L I O G R A F I A}

[1] WALz, K.: Wie werden betontechnische Erkenntnisse für das Bauen nutzbar gemacht? beton 10 (1960) H. 10, S. 483/490; ebenso Betontechnische Berichte 1960, Beton-Verlag, Düsseldorf 1961, S. $107 / 125$.

[2] Walz, K, und G. WIschers: Zum Entwurf der Neufassung der Zementnorm DIN 1164 , beton 18 (1968) H. 1, S. 10/14; ebenso Betontechnische Berichte 1968, Beton-Verlag, Düsseldorf 1969, S. 21/32.

[3] Wischers, G.: Einflu $\beta$ einer Temperaturänderung auf die Festigkeit von Zementstein und Zementmörtel mit Zuschlagstoffen verschiedener Wärmedehnung. Schriftenreihe der Zementindustrie, H. 28, Düsseldorf 1961, S. 52 und S. 53; ferner Wischers, G.: Physikalische Eigenschaften des Zementsteins. beton 11 (1961) H. 7, S. 481/486, Abschnitt 4, ebenso Betontechnische Berichte 1961, BetonVerlag, Düsseldorf 1962, S. 199/213.

[4] Locher, F. W.: Stöchiometrie der Hydratation von Tricalciumsilicat. Zement-Kalk-Gips 20 (1967) H. 9, S. $402 / 407$.

[5] Darms, J.: Einfluß der Eigenfeuchtigkeit auf die Druckfestigkeit des Betons. beton 18 (1968) H. 9, S. 361/365; ebenso Betontechnische Berichte 1968, Beton-Verlag, Düsseldorf 1969, S. $113 / 126$.

[6] ACI-Committee 211: Proposed revision of ACI 613-54. Proc. Amer. Concr. Inst. 66 (1969) S. 612/628.

[7] WALZ, K.: Beton- und Zementdruckfestigkeiten in den USA und ihre Umrechnung auf deutsche Prüfwerte. beton 12 (1962) H. 9, S. 420/423, und H. 10, S. 463/466; ebenso Betontechnische Berichte 1962, Beton-Verlag, Düsseldorf 1963, S. 123/140.

[8] Walz, K.: Anleitung für die Zusammensetzung von Beton mit bestimmten Eigenschaften. 2. Aufl., Verlag von Wilhelm Ernst \& Sohn, Berlin-München 1963.

[9] Meyer, A.: Über den Einfluß des Wasserzementwertes auf die Frühfestigkeit von Beton. BetonsteinZeitung 29 (1963) H. 8, S. 391/394 (Tafeln 1 u. 5).

[10] BonzeL, J. und J. Dahms: Der Einfluß des Zements, des Wasserzementwerts und der Lagerung auf die Festigkeitsentwicklung des Betons. beton 16 (1966) H. 7, S. 299/305, und H. 8, S. 341/342; ebenso Betontechnische Berichte 1966, Beton-Verlag, Düsseldorf 1967, S. 115/138. 